$01,07,13$

\title{
Базовые элементы структуры границ зерен наклона. Часть І. Ось разориентации [100]
}

\author{
(C) А.В. Векман, Б.Ф. Демьянов \\ Алтайский государственный технический университет им. И.И. Ползунова, \\ Барнаул, Россия \\ E-mail: weckman@list.ru
}

Поступила в Редакцию 26 февраля 2020 г.

В окончательной редакции 12 марта 2020 г.

Принята к публикации 6 августа 2020 г.

\begin{abstract}
Методами компьютерного моделирования проведен расчет структуры и энергии симметричных границ зерен наклона с осью разориентации [100]. Расчеты проводились с использованием структурно-вакансионной модели, ранее разработанной авторами. Углы разориентации границ зерен общего типа варьировались от $0^{\circ}$ до $90^{\circ}$, а шаг составил $1^{\circ}$. Специальные границы зерен имели обратную плотность совпадающих узлов $\Sigma \leq 53$. Расчет проводился с использованием парного потенциала Морзе и многочастичного потенциала Клери-Розато. Показано, что зависимость энергии границ зерен от угла разориентации при расчете разными потенциалами имеет похожий вид, а их структура не зависит от выбора потенциала и хорошо согласуется с электронно-микроскопическими изображениями высокого разрешения. На энергетической кривой выделенной является только одна специальная граница зерен $-\Sigma 5(013)$. Обнаружено, что структура границ зерен может быть представлена ограниченным числом групп атомов, которые были названы базовыми структурными элементами. Структура малоугловых границ зерен с углом разориентации менее $8^{\circ}$ описывается чередованием базового структурного элемента типа $D$ и идеального кристалла, от $8^{\circ}$ до $13^{\circ}-$ $C$ и $D$, от $14^{\circ}$ до $23^{\circ}-B$ и $C$. Большеугловые границы в диапазоне углов разориентации от $24^{\circ}$ до $37^{\circ}$ содержат только элементы тип $B$, от $38^{\circ}$ до $50^{\circ}-A$ и $B$, больше $50^{\circ}$ - только $A$.
\end{abstract}

Ключевые слова: компьютерное моделирование, граница зерен, структурно-вакансионная модель, базовые структурные элементы.

DOI: 10.21883/FTT.2020.12.50201.397

\section{1. Введение}

Границы зерен (ГЗ) являются одним из основных элементов дефектной структуры поликристаллов. Первые исследования представляли границу как бесструктурную аморфную область. Развитие методов исследования привело к тому, что в 1949 г. была предложена модель решетки совпадающих узлов (РСУ) [1]. Эта модель дала возможность ввести понятие границ специального типа, a eе развитие привело к модели структурных единиц (CE) [2]. Хотя авторы не называли свою модель моделью структурных единиц, в этой работе предложены основные ее принципы, сформулированные на примере границ с осью разориентации [100]. Построив в модели РСУ границу $\Sigma 17$ и две ГЗ $\Sigma 5$ с углами разориентации $28.1^{\circ}, 36.9^{\circ}$ и $53.1^{\circ}$ соответственно, авторы обнаружили повторяющиеся группы атомов в их структуре и обозначили их символами „4“, „3“ и „2“. Такое обозначение является вполне логичным, поскольку указывает на плоскость залегания этих ГЗ (014), (013) и (012) соответственно. Граница $\Sigma 13$ с углом разориентации $22.6^{\circ}$ так же рассмотренная в этой работе и получается из модели РСУ заменой двух самых сближенных атомов одним. Таким образом, структурный элемент этой ГЗ получается добавлением атома в плоскости границы к элементу „4“. Произвольные ГЗ с углами разориентации $\Theta^{\circ}<28.1^{\circ}$ рассматриваются как чередование участков идеального кристалла и элементов „4“. Авторы выделяют еще три диапазона углов разориентации: $28.1^{\circ}<\Theta<36.9^{\circ}, 36.9^{\circ}<\Theta<53.1^{\circ}$ и $\Theta>53.1^{\circ}$. В этих диапазонах произвольные границы представляются набором элементов „4“" и „,3“, „3“ и „2“, „2“ и идеального кристалла соответственно. Для примера в работе рассмотрены структуры симметричных границ с углами разориентации $61.9^{\circ}, 67.4^{\circ}$, $73.7^{\circ}$ и $79.6^{\circ}$, которые имели структуры $-|212| 212 \mid-$, -|21|21|-, -|121|121|- и -|11211|11211|-, где символом „1“ обозначены участки идеального кристалла. Авторы также утверждали, что данный принцип можно распространить и на другие типы границ.

Серия работ [3-5] опубликованных Саттоном и Витеком в 1983 г. посвящена изучению структуры ГЗ с точки зрения модели СЕ. В первой [3] рассмотрена структура симметричных ГЗ с осями разориентации [100], [110] и [111], вторая [4] посвящена несимметричным границам, а третья [5] обобщению результатов и анализу влияния структуры границ на их свойства. Для симметричных ГЗ с осью [100] рассмотрено пять специальных границ $\Sigma 1, \Sigma 41, \Sigma 37, \Sigma 17$ и $\Sigma 5$ с углами разориентации $0^{\circ}, 12.68^{\circ}, 18.92^{\circ}, 28.87^{\circ}$ и $36.87^{\circ}$. Параметры потенциала межатомного взаимодействия при моделирования соответствовали меди. Граница $\Sigma 1$ 
представляет собой идеальный кристалл, структурный элемент которой был обозначен символом „, $A^{“,}$, а $\Sigma 5-$ состоит из элементов типа „В“. Структура остальных границ: $\Sigma 41-A A A B B . A A A B ; \Sigma 37-A A B A B$ и $\Sigma 17-A B B$, т.е. все промежуточные по углам границы состоят из элементов границ $\Sigma 1$ и $\Sigma 5$, которые были названы предпочтительными.

Более подробное описание структуры границ с осью разориентации [100] можно найти в работах [6,7]. В этих работах на примере меди рассмотрена структура границ во всем диапазоне углов разориентации. Как и в [3] структурные элементы идеального кристалла (в работе обозначенного границей $\Sigma 1$ ) названы структурными единицами типа „A“. Для границы $\Sigma 5$ с углом разориентации $36.87^{\circ}$ обнаружены две структурные единицы соответствующая модели РСУ (названа „B“) и содержащая одну вакансию на структурную единицу (названа

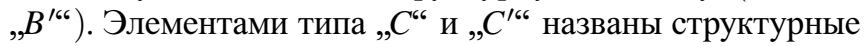
единицы ГЗ $\Sigma 5$ с углом разориентации $53.13^{\circ}$, также совпадающие со структурой в модели РСУ и с одной вакансией соответственно. Элемент типа „D“ соответствует идеальному кристаллу, полученному поворотом каждой половинки бикристалла на угол $45^{\circ}$. Таким образом, авторы выделяют три диапазона углов $0^{\circ}-36.87^{\circ}$, $36.87^{\circ}-53.13^{\circ}$ и $53.13^{\circ}-90^{\circ}$ в которых любые границы состоят из элементов: $A, B$ и $B^{\prime} ; B, B^{\prime}, C$ и $C^{\prime} ; C$, $C^{\prime}$ и $D$, причем стабильные структуры границ могут содержать от одной до восьми модификаций с разным чередованием структурных элементов. Примечательно, что энергии различных модификаций структур могут иметь как близкие значения, так и значительно отличаться друг от друга. Например, границы $\Sigma 41$ и $\Sigma 13$ имеют разброс энергий менее $0.5 \%$, тогда как для ГЗ $\Sigma 5, \Sigma 17$ и $\Sigma 73$ - более $17 \%$. В [8] моделировались Г3 с осью разориентации [100] в $\mathrm{Ni}, \mathrm{Al}$ и $\mathrm{Ni}_{3} \mathrm{Al}$. Результаты, полученные в этой работе, согласуются с результатами исследований, приведенными в работах $[6,7]$.

Применение модели СЕ встречается не только при моделировании ГЗ в металлах. Например, в работах $[9,10]$ изучается структура симметричных ГЗ с осью разориентации [100] в кремнии. Авторы выделяют четыре структуры, названные элементами $A, A^{\prime}, B$ и $C$. Исследована структура 18-ти границ. Границы с углами разориентации $0^{\circ}-53.13^{\circ}$ состоят из элементов $A, A^{\prime}$ и $B$, а $53.13^{\circ}-90^{\circ}-A$ и $C$. Сама граница $\Sigma 5$ описана либо элементами $B$, либо $C$.

Есть и более поздние работы использующие принципы, заложенные в [2]. Например, авторы [11] провели моделирование ГЗ с использованием дисклинационной модели. Изучались ГЗ в меди и никеле. В отличие от работ Саттона и Витека авторы обнаружили, что структура типа $B$ неустойчива и всегда релаксирует к структуре $B^{\prime}$. В остальном данное исследование совпадает с результатами ранее проведенных расчетов. В работе [12] поиск структурных единиц проведен в вольфраме. Показано, что для оси [100] в вольфраме существует только четыре короткопериодических ГЗ.
Несмотря на широкое применение возможности модели СЕ для прогнозирования свойств ГЗ остаются ограниченными, что связано с множественностью структур на атомном уровне [13]. В работе [14] предложен общий подход, в котором ГЗ рассматривается как дефект, имеющий фазовые переходы, при которых их структура и свойства могут изменяться прерывисто при критических значениях термодинамических параметров, таких как температура, давление и химический потенциал. Сама атомная структура при этом не анализируется. А в работах $[15,16]$ изменение структурных единиц рассматриваются как фазовые переходы на ГЗ.

Таким образом, хотя большинство работ проведенных в рамках модели СЕ показывают совпадающие результаты, имеются разночтения в строении границ полученных разными авторами. Целью настоящей работы является исследование атомного строения ГЗ наклона с осью разориентации [100], выявление индивидуальных особенностей и общих закономерностей строения границ в рамках модели $\mathrm{CE}$.

\section{2. Модель}

Исследование проведено с использованием разработанной ранее структурно-вакансионной модели ГЗ. Основные положения модели изложены в работах $[17,18]$. Данная модель позволяет определять атомную структуру ГЗ наклона общего и специального типа. В основе используемой модели лежит потенциальная энергия атомов. Исходная конфигурация атомов выбирается в модели РСУ. При этом часть атомов в области ГЗ находятся на расстоянии, отличающемся от равновесного, в результате чего граница в модели РСУ имеет высокое значение энергии. Введение дополнительных атомов и вакансий стабилизирует структуру границы и значительно понижает ее энергию. Данная процедура называется вакансионной релаксацией $[19,20]$. После вакансионной релаксации проводится смещение атомов под действием межатомных сил, которое дополнительно понижает энергию ГЗ и стабилизирует ее структуру. Расчеты проводились с использованием парного потенциала Морзе [21] и многочастичного потенциала КлериРозато [22]. Параметры потенциалов соответствовали алюминию. Сравнение данных, полученных при использовании двух разных потенциалов, показало, что структура ГЗ не зависит от выбора вида потенциала $[18,23]$.

На рис. 1 представлена зависимость энергии стабильных ГЗ с осью разориентации [100]. Были рассчитаны структура и энергия границ общего типа во всем интервале углов разориентации $\Theta$. Шаг по углам разориентации составил один градус. Специальные ГЗ отмечены на рисунке маркерами. Цифрами обозначена обратная плотность совпадающих узлов этих границ в соответствии с моделью РСУ. Параметры специальных ГЗ, рассчитанных в работе, приведены в таблице. 


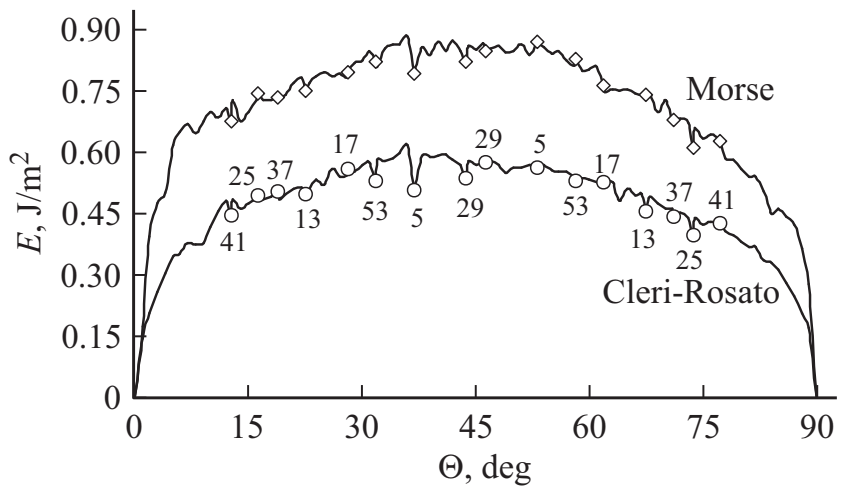

Рис. 1. Зависимость энергии ГЗ от угла разориентации.

Как видно из рис. 1 зависимости имеют подобный вид, отсутствуют глубокие провалы энергии для специальных ГЗ, выделенными на энергетической кривой является только граница $\Sigma 5(013)$. Кривая, рассчитанная с использованием потенциала Морзе, дает более высокие значения энергии по сравнению с потенциалом Клери-Розато. Величина энергии большеугловых ГЗ, полученная с использованием потенциала Морзе лежит в диапазоне $0.70-0.85 \mathrm{~J} / \mathrm{m}^{2}$, а полученная с использованием потенциала Клери-Розато - 0.45-0.55 J/m². Экспериментальное значение энергии ГЗ для алюминия составляет $0.60 \mathrm{~J} / \mathrm{m}^{2}$ [24], т. е. энергия, полученная с применением парного потенциала, является завышенной, а многочастичного - заниженной.

\section{3. Результаты и их обсуждение}

Так как каждая ГЗ специального типа состоит из набора одинаковых структурных элементов, то логично будет начать рассмотрение со специальных границ. Начнем с двух наиболее изученных границ $\Sigma 5(012)$ и $\Sigma 5(013)$. Структурные элементы этих ГЗ в нашей модели представлены на рис. 2. Большими кругами обозначены атомы ближней к наблюдателю плоскости, а маленькими - следующей за ней плоскости, которая находится на расстоянии $a / 2$ ( $a$ - параметр решетки). Каждый элемент данных границ может быть
Параметры специальных ГЗ: обратная плотность совпадающих узлов $(\Sigma)$; индексы Миллера плоскости ГЗ $(h k l)$ и угол разориентации $(\Theta)$

\begin{tabular}{|c|c|c|c|c|c|}
\hline$\Sigma$ & $h k l$ & $\Theta,,^{\circ}$ & $\Sigma$ & $h k l$ & $\Theta,^{\circ}$ \\
\hline 5 & 013 & 36.87 & 5 & 012 & 53.13 \\
\hline 13 & 015 & 22.62 & 13 & 023 & 67.38 \\
\hline 17 & 014 & 28.07 & 17 & 035 & 61.93 \\
\hline 25 & 017 & 16.26 & 25 & 034 & 73.74 \\
\hline 29 & 025 & 43.60 & 29 & 037 & 46.40 \\
\hline 37 & 016 & 18.92 & 37 & 057 & 71.08 \\
\hline 41 & $\begin{array}{lll}0 & 19\end{array}$ & 12.68 & 41 & 045 & 77.32 \\
\hline 53 & 027 & 31.89 & 53 & 059 & 58.11 \\
\hline
\end{tabular}

представлен в виде набора групп атомов, выделенных линиями разного типа. Эти группы атомов мы назвали базовыми структурными элементами типа $A$ (рис. $2, a$ ) и $B$ (рис. $2, b)$. Серым цветом ограничены структурные элементы границ в модели РСУ, а стрелка означает то, что элемент смещен на одну плоскость от наблюдателя. Объемный вид этих групп атомов представлен на рис. 2, $c$ и рис. $2, d$ соответственно. Данные структурные элементы получаются из структуры РСУ при удалении двух атомов на одни период повторяемости.

Как можно видеть из рис. 1 две специальные границы $\Sigma 29(025)$ и $\Sigma 29(037)$ лежат на энергетической кривой между границами $\Sigma 5(012)$ и $\Sigma 5(013)$. В соответствии с моделью СЕ данные границы могут иметь собственные структурные элементы, либо состоять из элементов границ представленных на рис. 2. На рис. 3 представлены структурные элементы этих границ. Граница $\Sigma 29(025)$ состоит из строго чередующихся базовых структурных элементов типа $A$ и $B$, расстояние между которыми примерно равно радиусу первой координационной сферы. В структуре границы $\Sigma 29(037)$ наряду с элементами типа $A$ и $B$ присутствует группа атомов, которая совпадает со структурой границы $\Sigma 5(012)$ в модели РСУ. Ее объемный вид представлен на рис. $3, c$.

Рассмотрим структуру специальных ГЗ со значениями угла разориентации больше, чем у границы $\Sigma 5(012)$. Их периоды представлены на рис. 4. На рисунке границы расположены в порядке возрастания их углов $\Theta$ (см. таблицу). При внимательном изучении структуры этих
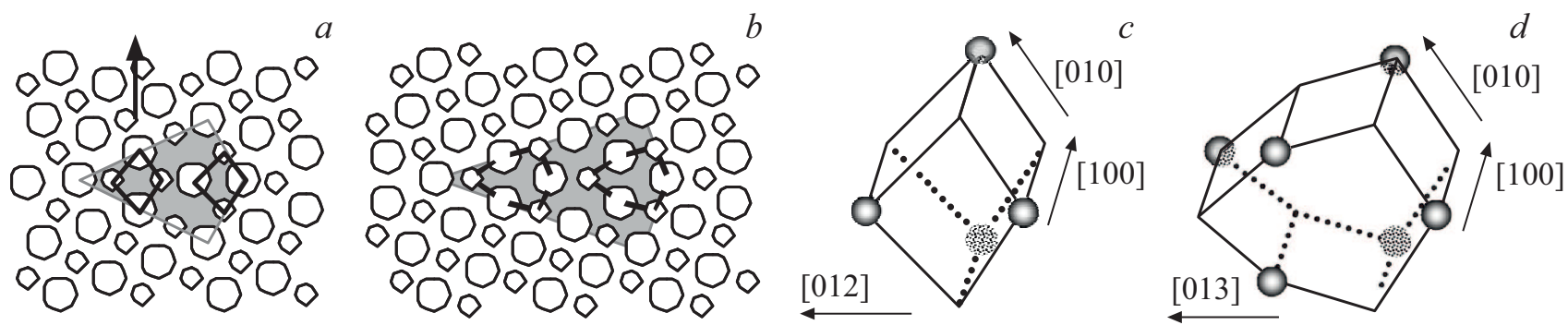

Рис. 2. Структурные элементы специальных ГЗ $\Sigma 5(012)(a)$ и $\Sigma 5(013)(b)$ и базовые структурные элементы типа $A(c)$ и типа $B(d)$. 

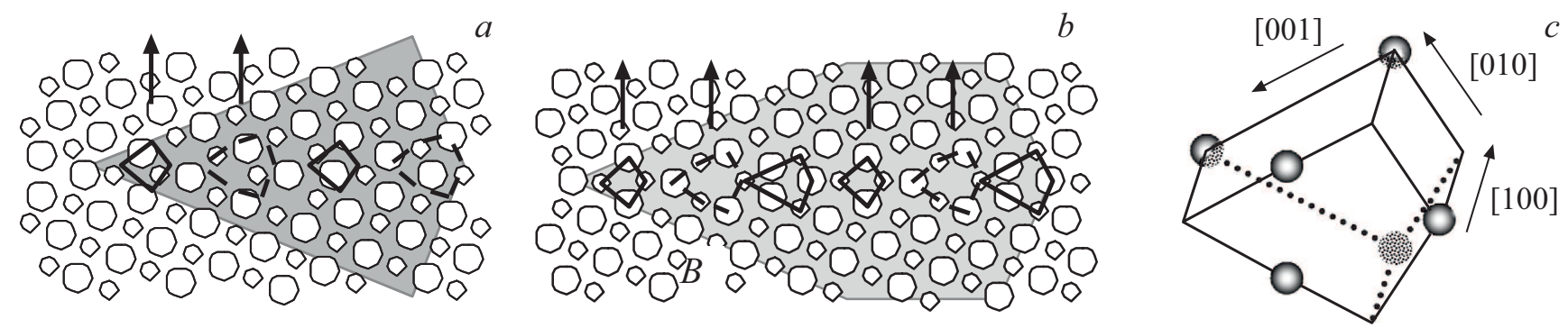

Рис. 3. Структурные элементы специальных ГЗ (a) $\Sigma 29(025)$ (а) и $\Sigma 29(037)(b)$ и РСУ модификация базового структурного элемента типа $A(c)$.

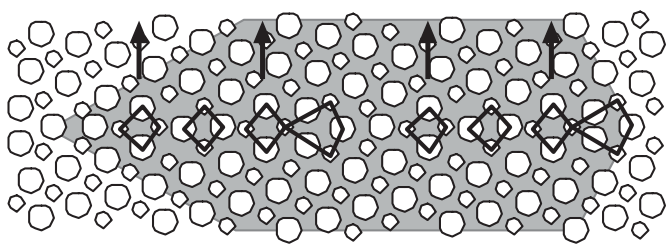

$\Sigma 53(059)$

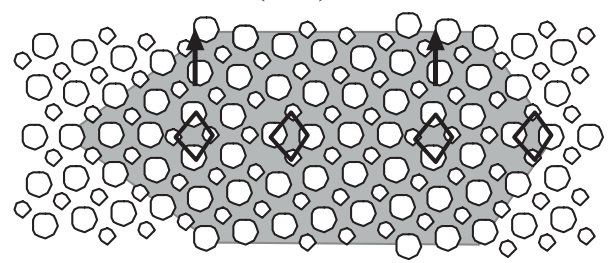

$\Sigma 37(057)$

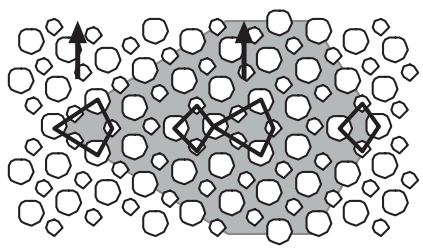

$\Sigma 17(035)$

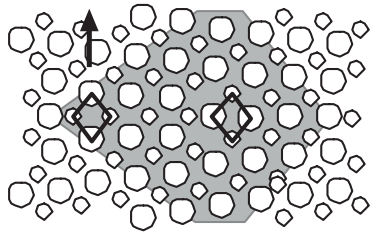

$\Sigma 25(034)$

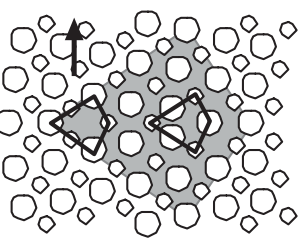

$\Sigma 13(023)$

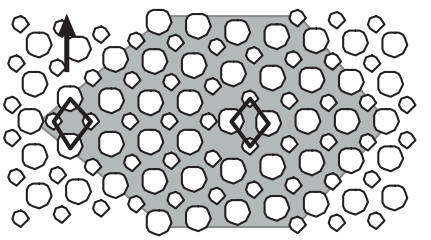

$\Sigma 41(045)$

Pис. 4. Структурные элементы специальных ГЗ с углами разориентации больше, чем у $\Sigma 5(012)$.

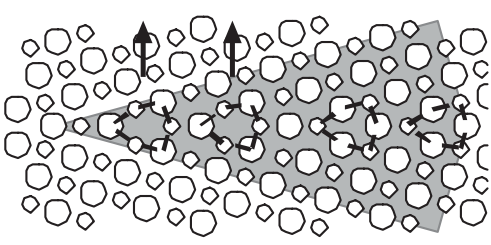

$\Sigma 53(027)$

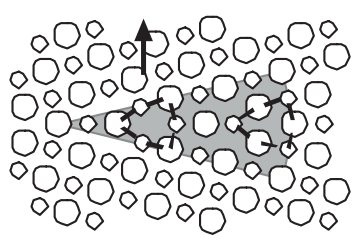

$\Sigma 17(014)$

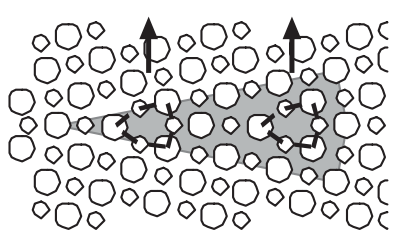

$\Sigma 13(015)$

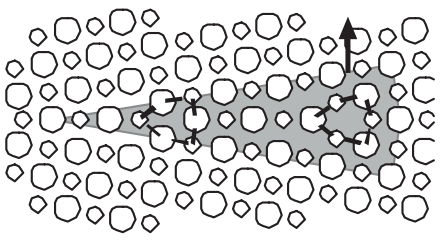

$\Sigma 37(016)$

Рис. 5. Структурные элементы специальных ГЗ с углами разориентации меньше, чем у $\Sigma 5(013)$.

границ были обнаружены две особенности. Во-первых, все границы составлены из структурных элементов типа $A$ и его РСУ модификации, т. е. структурных элементов границы $\Sigma 5(012)$. Во-вторых, по мере увеличения угла разориентации $\Theta$, расстояние между структурными элементами растет, а область бикристалла между ними представляет собой почти идеальный кристалл. Анализ границ общего типа показал, что все границы с $\Theta \geq 54^{\circ}$ тоже состоят из этих элементов. Эти особенности являются вполне логичными. При развороте кристалла вокруг оси разориентации граница образуется сочленением плоскостей типа (001). Угол между этими плоскостями и является углом $\Theta$. При проведении вакансионной релаксации часть из атомов удаляется из области ГЗ что приводит к образованию распределенных вакансий [18] и, следовательно, возникают элементы типа $A$. Там где возникновение распределенной вакансии оказывается энергетически не выгодным, образуется элемент типа РСУ модификация элемента $A$. Промежутки между структурными элементами заполняются участками почти идеального кристалла. При увеличении угла разориентации границу можно рассматривать как структуру образованную сочленением плоскостей (110) под малыми углами. При увеличении угла $\Theta$ угол между этими плоскостями уменьшается, что и приводит к образованию участков идеального кристалла. 
$a$

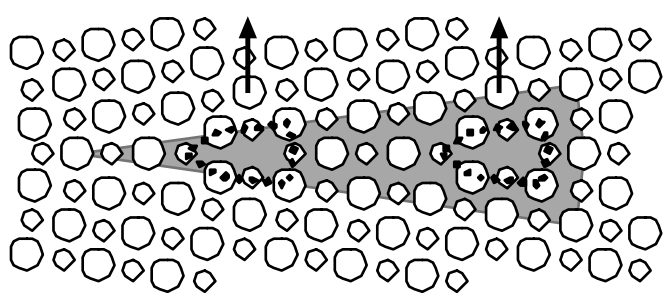

$b$

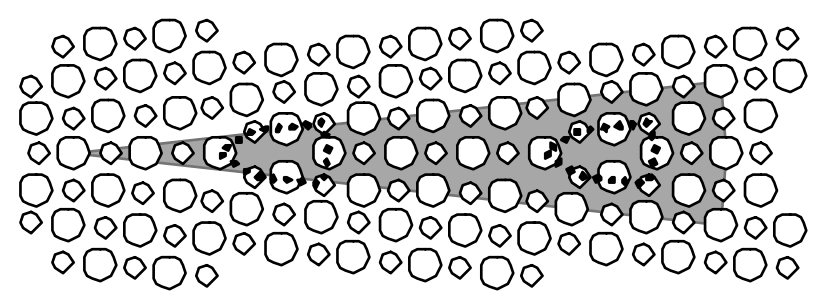

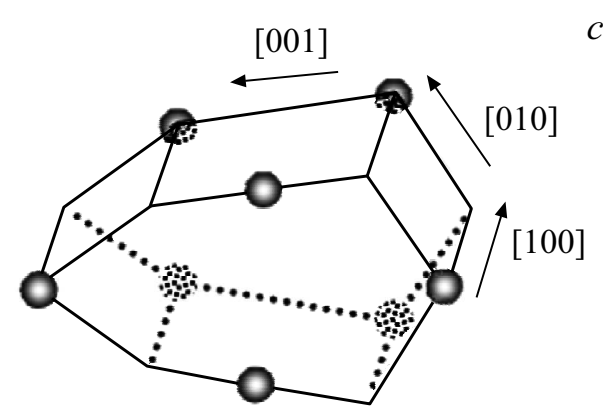

$c$

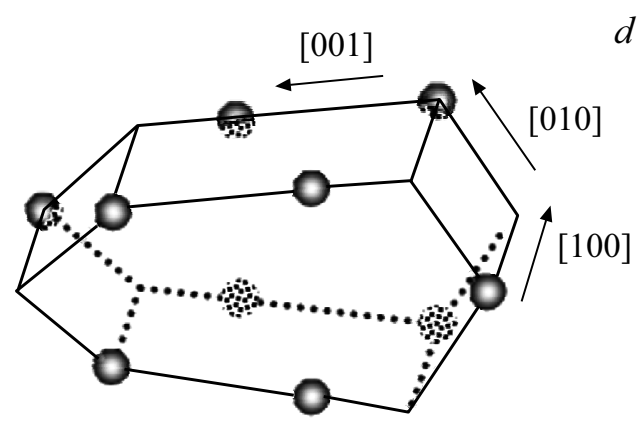

Рис. 6. Структурные элементы специальных ГЗ с малыми углами разориентации $\Sigma 25(017)(a)$ и $\Sigma 41(019)$ (b). Базовые структурные элементы ГЗ типа $C(c)$ и типа $D(d)$.

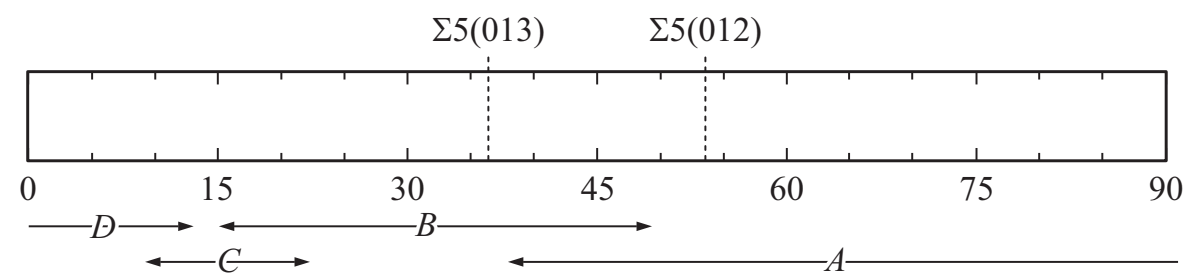

Рис. 7. „Шкала“ базовых структурных элементов ГЗ с осью разориентации [100].

Структура специальных ГЗ с углами разориентации меньше, чем у $\Sigma 5(013)$ имеет вид, представленный на рис. 5. Границы представлены в порядке уменьшения их углов разориентации (см. таблицу). Как и в случае границ с углами $\Theta \geq 53.13^{\circ}$ наблюдаются две особенности: границы составлены из структурных элементов типа $B$, т.е. структурных элементов границы $\Sigma 5(013)$ и по мере уменьшения угла разориентации, расстояние между структурными элементами растет, а область между ними представляет собой почти идеальный кристалл. Анализ границ общего типа показал, что структурные элементы типа $B$ встречаются в структурах с $15^{\circ} \leq \Theta \leq 50^{\circ}$.

Такая структура большеугловых границ объясняется тем, что при уменьшении угла разориентации с одной стороны все больше атомов плоскостей (001) сближается друг с другом, а с другой образуются области с значительным избыточным объемом. В процессе вакансионной релаксации образовавшиеся полости заполняются дополнительными атомами, а один из сблизившихся удаляется. Например, ГЗ $\Sigma 53$ и $\Sigma 17$ содержат по шесть и четыре распределенные вакансии соответственно. В границах $\Sigma 13$ и $\Sigma 27$ наряду с распре- деленными вакансиями, число которых четыре и шесть соответственно, на период повторяемости добавляется по два дополнительных атома.

Еще две специальные ГЗ $\Sigma 25$ и $\Sigma 41$ можно отнести к малоугловым, т.к. их углы разориентации равны $6.26^{\circ}$ и $12.68^{\circ}$ соответственно. Базовый структурный элемент этих границ отличается от базовых структурных элементов большеугловых ГЗ. На рис. $6, a$ и $b$ изображены периоды повторяемости данных границ, а на рис. 6, $c$ объемный вид структурного элемента этих ГЗ, обозначенный нами символом „С“. Как и в случае большеугловых границ с уменьшением угла разориентации увеличивается расстояние между базовыми элементами.

Анализ структуры малоугловых ГЗ общего типа показал, что постепенная смена структурных элементов с типа $B$ на тип $C$ происходит, когда угол разориентации становится меньше $23^{\circ}$. А в границах с углами разориентации меньше $15^{\circ}$ элемент типа $B$ не встречается. Наряду с структурными элементами типа $C$ в малоугловых ГЗ общего типа присутствуют так же элементы типа $D$, объемный вид которых представлен на рис. $6, d$, причем с уменьшением угла $\Theta$ элементов типа $C$ становится 
все меньше, а элементов типа $D$ больше. Минимальный угол разориентации в котором было отмечено наличие структурных элементов $C$ был равен $8^{\circ}$.

Таким образом, структура всей совокупности ГЗ с осью разориентации [100] состоит всего из четырех базовых структурных элементов. На рис. 7 представлена „шкала“ базовых структурных элементов. Эта „шкала“ показывает в пределах каких углов разориентации границы зерен содержат базовые структурные элементы определенного типа.

\section{4. Заключение}

В рамках структурно-вакансионной модели рассчитана атомная структура ГЗ наклона с осью разориентации [100]. Изучались границы общего и специального типов, углы разориентации которых варьировался по полному угловому диапазону - от $0^{\circ}$ до $90^{\circ}$. При расчете энергии ГЗ использовались парный потенциал Морзе и многочастичный потенциал Клери-Розато. Структуры ГЗ, полученные с использованием этих потенциалов, совпадают с высокой точностью. Зависимости энергии ГЗ от угла разориентации имеют подобный характер. Энергия, рассчитанная с использованием потенциала Морзе, завышена относительно экспериментального значения, а рассчитанная с использованием потенциала Клери-Розато, напротив - занижена.

Анализ атомной структуры позволил выделить базовые структурные элементы, из которых построены все симметричные ГЗ наклона, как общего, так и специального типов. Исследованы закономерности присутствия базовых структурных элементов в зависимости от угла разориентации. Специальные ГЗ $\Sigma 5(012)$ и $\Sigma 5(013)$ состоят из базовых структурных элементов одного типа $A$ и $B$ соответственно. Большеугловые ГЗ специального и общего типов $(\Theta>20)$ состоят из комбинации базовых структурных элементов $A$ и $B$ в определенном соотношении. Малоугловые ГЗ специального и общего типов состоят из базовых структурных элементов $C$ и $D$. Участки границ между базовыми структурными элементами представляют собой почти идеальный кристалл. По мере приближения угла разориентации к углам $0^{\circ}$ и $90^{\circ}$ протяженность этих участков возрастает.

\section{Конфликт интересов}

Авторы заявляют, что у них отсутствует конфликт интересов.

\section{Список литературы}

[1] M.L. Kronberg, F.N. Wilson. Trans. AIME 185, 506 (1949).

[2] G.H. Bishop, B. Chalmers. Scripta Met. 2, 133 (1968).

[3] A.P. Sutton, V. Vitek. Phil. Trans. R. Soc. Lond. A 309, 1 (1983).

[4] A.P. Sutton, V. Vitek. Phil. Trans. R. Soc. Lond. A 309, 37 (1983).
[5] A.P. Sutton, V. Vitek. Phil. Trans. R. Soc. Lond. A 309, 55 (1983).

[6] V. Vitek, A.P. Sutton, G.-J. Wang, D. Schwartz. Scr. Met. 17, 183 (1983).

[7] G.-J. Wang, A.P. Sutton, V. Vitek. Acta Met. 32, 1093 (1984).

[8] S.P. Chen, D.J. Srolovitz, A.F. Voter. J. Mater. Res. 4, 62 (1989).

[9] A.A. Levi, D.A. Smith, J.T. Wetzel. J. Appl. Phys. 69, 2048 (1991).

[10] D.A. Smith. Interface Sci. 4, 11 (1997).

[11] Д.В. Бачурин, Р.Т. Мурзаев, А.А. Назаров. ФММ 96, 11 (2003).

[12] J. Han, V. Vitek, D.J. Srolovitz. Acta Mater. 133, 186 (2017).

[13] N.J. Burbery, R. Das, W.G. Ferguson. Mater. Lett. 158, 413 (2015).

[14] P.R. Cantwell, M. Tang, S.J. Dillon, J. Luo, G.S. Rohrer, M.P. Harmer. Acta Mater. 62, 1 (2014).

[15] T. Frolov, D.L. Olmsted, M. Asta, Y. Mishin. Nat. Comm. 4, 1899 (2013).

[16] T. Frolov, M. Asta, Y. Mishin. Phys. Rev. B 92, 020103 (2015).

[17] А.С. Драгунов, А.В. Векман, Б.Ф. Демьянов. Ползуновский альманах 4-1, 29 (2011).

[18] А.В. Векман, Б.Ф. Демьянов. ФММ 120, 53 (2019).

[19] M.D. Starostenkov, B.F. Demyanov, A.V. Weckman. Acta Metallurgica Sinica (English Lett.) 13, 540 (2000).

[20] А.С. Драгунов, Б.Ф. Демьянов, А.В. Векман. Изв. вузов. Физика 53, 82 (2010).

[21] P.M. Morse. Phys. Rev. 34, 57 (1929).

[22] F. Cleri, V. Rosato. Phys. Rev. B 48, 22 (1993).

[23] А.В. Векман, Б.Ф. Демьянов, А.С. Драгунов. Изв. АлтГУ 1, 99, 11 (2018).

[24] Дж. Хирт, И. Лоте. Теория дислокаций. Атомиздат, М. (1972). $600 \mathrm{c}$.

Редактор Т.Н. Василевская 